\title{
Genetics and general cognitive ability
}

\author{
Robert Plomin
}

General cognitive ability ( $g$ ), often referred to as 'general intelligence', predicts social outcomes such as educational and occupational levels far better than any other behavioural trait. $g$ is one of the most heritable behavioural traits, and genes that contribute to the heritability of $g$ will certainly be identified. What are the scientific and social implications of finding genes associated with $g$ ?

$\mathrm{D}$ uring the past three decades, the behavioural sciences have emerged from an era of strict environmental explanations for differences in behaviour to a more balanced view that recognizes the importance of nature (genetics) as well as nurture (environment). This shift occurred first for behavioural disorders, including rare disorders such as autism (which has an incidence of 1 per 1,000 population), more common disorders such as schizophrenia (1 in 100), and very common disorders such as reading disability ( 1 in 50). More recently, it has become increasingly accepted that genetic variation makes an important contribution to differences among individuals in the normal range of behaviour as well as for abnormal behaviour. Moreover, many behavioural disorders, especially common ones, may represent variation at the extremes of the same genetic and environmental factors that are responsible for variation within the normal range. For example, disorders such as reading disability may not be due to genetic variants that specifically influence the disorder. Rather, the same genes that contribute to the normal range of individual differences in reading ability may be responsible for reading disability (Fig. 1). This view, known as the quantitative trait locus (QTL) perspective (see Box 1), has important implications for the search for the genes responsible for behaviour because such genes will individually have small effects; this will make them more difficult to find than genes that have major effects ${ }^{1}$.

\section{General cognitive ability}

For historical and political reasons, one quantitative trait in particular is highly controversial. This is general cognitive ability, which has a normal distribution in the population from a low end of mild mental handicap to a high end of gifted individuals. Diverse measures of cognitive abilities — such as spatial ability, verbal ability, information processing speed and

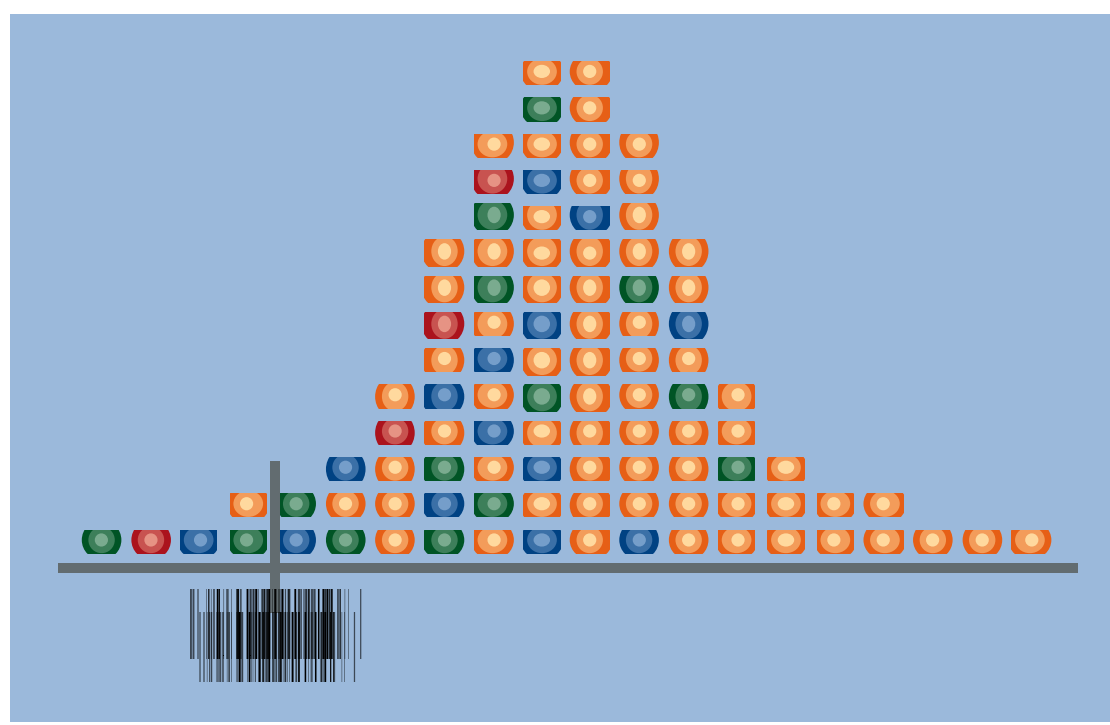

Figure 1 Quantitative trait locus (QTL) perspective on complex traits. Differences among individuals for most quantitative or complex traits such as reading ability are distributed as a normal bell-shaped curve. Multiple genes influence complex traits as probabilistic propensities rather than predetermined programmes. Here the different genetic make-up of individuals with respect to two hypothetical genes involved in reading ability is shown for 100 individuals (each person is represented by an oval), with five of these individuals (those on the extreme left) receiving a diagnosis of reading disability. The green ovals indicate that the individual has the disabling variant of one gene and blue ovals denote the disabling variant of the other gene. Neither gene is necessary or sufficient for low scores, even for individuals who have disabling variants of both genes (red ovals). This QTL perspective suggests that genes associated with common disorders such as reading disability may represent the quantitative extreme of the same genes that are responsible for variation throughout the population.

memory - correlate substantially with each other, and general cognitive ability $(g)$ is what these diverse measures have in common (see Box 2). Clearly there is more to cognition than $g$ - although $g$ explains about 40 per cent of the variance among such tests, most of the variance of a particular test is independent of $g$.

There is a wide gap between what lay people (including scientists in other fields) believe about intelligence and intelligence testing, and what the professional behavioural scientist believes. Most notably, lay people often read in the popular press that the assessment of intelligence is circular — intelligence is what intelligence tests assess. On the contrary, $g$ is one of the most reliable and valid measures in the behavioural domain; its long-term stability after childhood is greater than for any other behavioural trait, and it predicts important social outcomes such as educational and occupational levels far better than any other trait $^{2}$. Although a few critics remain ${ }^{3}, g$ is widely accepted by experts ${ }^{4}$. But it is less clear what $g$ is: is it due to a single general process 


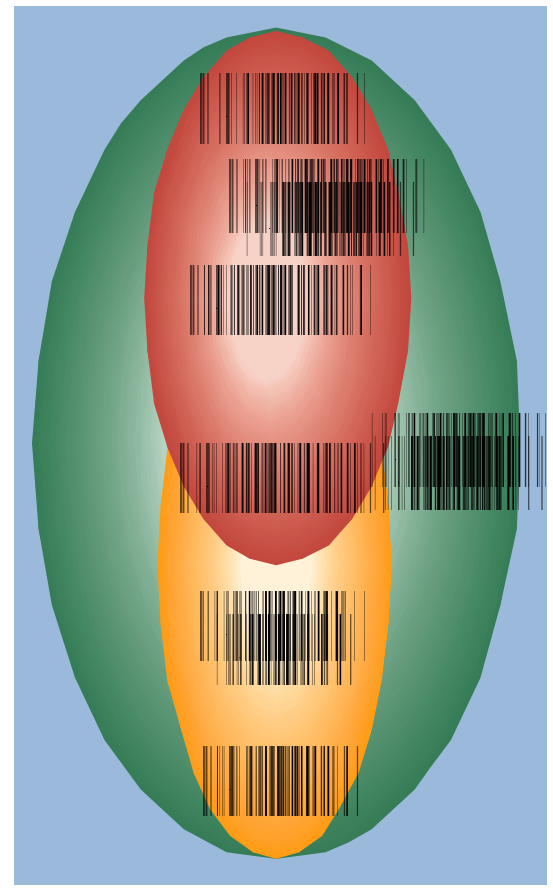

Figure 2 Functional genomics includes all levels of analysis from molecular biology to psychology. The higher levels of analysis can be referred to as behavioural genomics in order to emphasize the importance of top-down analyses of pathways between genes and behaviour.

such as high-level strategies called executive function or speed of information processing, or does it represent a concatenation of more specific cognitive processes? ${ }^{5,6}$

The concept of a genetic contribution to $g$ has provoked much controversy, especially following the publication in 1994 of The Bell Curve by Herrnstein and Murray ${ }^{7}$. (This book in fact scarcely touches on genetics and does not view genetic evidence as crucial to its arguments.) The first half of the book shows, like many other studies, that $g$ is related to educational and social outcomes, but the second half attempts to argue that certain right-wing policies follow from these findings. However, as discussed later in this article, public policy does not necessarily follow from scientific findings, and it would be possible to argue in just the opposite direction from Herrnstein and Murray. Nonetheless, there is considerable consensus among scientists - even those who are not geneticists - that $g$ is substantially heritable ${ }^{6,8,9}$. Indeed, there are more studies addressing the genetics of $g$ than of any other human characteristic, including studies of more than 8,000 parent-offspring pairs, 25,000 pairs of siblings, 10,000 twin pairs and hundreds of adoptive families, all of which indicate that genetic factors contribute significantly to $g^{10,11}$.

Estimates of the size of the genetic effect, which population geneticists call 'heritabili- ty', vary from 40 to 80 per cent, but estimates based on the entire body of data make it about 50 per cent, indicating that genes account for about half of the variance in $g$. When the data are sorted by age, heritability is found to increase from about 20 per cent in infancy, to about 40 per cent in childhood, to 60 per cent or greater later in life ${ }^{12}$, even for individuals over 80 years old ${ }^{13}$. This increase in heritability throughout lifespan is interesting, because it is counterintuitive to the effects of Shakespeare's 'slings and arrows of outrageous fortune' accumulating over time. It may be that heritability increases because individuals seek out and create environments correlated with their genetic propensities.

Most of the genetic variance for $g$ is additive, that is, the effects of the individual genes seem simply to add up rather than there being interactions between the genes. The additivity of most genetic effects on $g$ may be because there is greater assortative mating (non-random mating) for $g$ than for any other behavioural trait. In other words, bright women are likely to mate with bright men and the outcome of this dual effect is that their offspring are likely to be brighter on average than would be expected if mating were at random, thus spreading out the distribution of $g$ in the population.

The data that provide evidence for a genetic effect on $g$ also provide the best available evidence for the importance of environmental factors that are independent of genetics. Environment clearly is important, as indicated by the steady rise in IQ scores during the past several generations, which would seem too short a time to be explained by genetics ${ }^{14}$, and by studies in which children from abusive families show gains in IQ when adopted ${ }^{15}$.

\section{Recent findings and new directions} Genetic research has moved beyond the rudimentary questions of whether, and to what extent, genetic differences are important in the origins of individual differences in $g$. These new findings inform the scientific and social implications discussed later.

It is often not appreciated that genetically sensitive designs, such as twin and adoption studies, that recognize the importance of both nature and nurture are uniquely well suited to the investigation of environmental influences. Indeed, one of the most important discoveries about environmental influences on $g$ has come from such genetic research. The 'nurture assumption'16 - that the home is the most important part of the child's environment - implies that children growing up in the same home should be similar to one another because they share these environmental influences. When genetic resemblance is taken into account, such shared environmental influences that contribute to the resemblance of family members for $g$ are important in childhood, accounting for about a quarter of the variance, but they are not important after adolescence. In other words, nonshared environmental factors that make children in the same family different (such as differences in parental treatment, differences in school experiences and different experiences with peers) provide the longterm consequences of environmental influence for $g$. This finding coincides with similar findings for other quantitative traits and indicates the need to re-examine the nurture assumption ${ }^{16}$.

Two other examples of recent genetic findings about the environment are that environmental influences may override genetic effects in families of low socioeconomic status ${ }^{17}$, and that genetic factors contribute to individuals' interactions with their environment ${ }^{18}$. The former finding highlights that heritability estimates are not absolute but depend on the environment in which they are measured. The latter observation, called 'genotype-environment correlation', indicates that genetic influences on abilities can best be thought of as

\section{Box1 The source of genetic variation}

Quantitative traits are those characteristics, such as height or 'intelligence', that are found as a continuum of values within a population rather than as the discrete alternative inherited character states familiar to most people from a schoolroom acquaintance with Mendel's peas. They are due to the combined effects of a number of different genes (each of which will, of course, be inherited according to the rules of mendelian genetics), and usually also have a considerable environmental input to the final outcome. This final outcome is known as the phenotype. A quantitative trait locus (QTL) refers to a gene that contributes to a quantitative trait. A locus is the technical name in genetics for the position on the chromosome at which a gene for a particular characteristic is located. In any outbreeding population, such as humans, many genes are present in the population in a number of variant forms (technically known as alleles). By inheritance from their mother and father each person carries two copies (that is, two alleles, which may be different or identical) of the genes corresponding to most loci (those on the sex chromosomes excepted). The genetic variation within the human population is due to the immense number of different combinations of alleles possible, given the tens of thousands of different loci in the human genome. These different combinations give each individual human being (except identical twins) a unique genetic make-up or genotype, even though all humans share the same set of loci. 
appetites rather than aptitudes in the sense that genetic propensities propel individuals towards evoking, selecting and constructing experiences that are correlated with their genetic propensities. Bright children select (and are selected by) peers and educational programmes that foster their abilities. They read and think more.

Work on genetic influences on intelligence has up to now focused on $g$. Much less is known about the genetic and environmental origins of individual differences in specific cognitive abilities such as spatial ability, verbal ability, memory and processing speed. Specific cognitive abilities show substantial genetic influence, although less than for $g^{19}$. To what extent do different sets of genes affect these different abilities? A technique called multivariate genetic analysis examines covariance among specific cognitive abilities and yields a statistic called the 'genetic correlation', which is the extent to which genetic effects on one trait correlate with genetic effects on another trait independently of the heritability of the two traits. That is, although individual cognitive abilities are moderately heritable, the genetic correlations between them could be anywhere from 0 , indicating that a completely different set of genes influences each ability, to 1, indicating that the same genes influence a variety of cognitive abilities. Multivariate genetic analyses have consistently found that genetic correlations among specific cognitive abilities are very high - close to 1 (ref. 20).

\section{Implications for cognition theory}

These results have major implications for current theories of how the brain works. According to one theory, the brain works in a modular fashion - the various cognitive processes are specific and independent of each other. Implicit in this perspective is a bottom-up reductionistic view of genetics in which individual modules such as spatial visualization are the targets of gene action. However, the findings from multivariate genetic analyses suggest a top-down view, in which genetic effects operate primarily on $g$. Given that the brain has evolved to learn from a variety of experiences and to solve a variety of problems, perhaps it makes sense that it would function holistically.

Nevertheless, finding genetic correlations near 1.0 does not prove that genetic effects are limited to a single general cognitive process. Another alternative is that specific cognitive abilities might tap many of the same modular processes that are each affected by different sets of genes. This hypothesis could be tested by multivariate genetic research on measures of modular processes, for example determining which areas of the brain are active in response to a particular task. Such procedures can now be done using neuroimaging techniques, and

\section{Box2 General cognitive ability $(g)$}

General cognitive ability $(g)$ is best captured by a technique called factor analysis in which a composite score is created that represents what diverse measures of cognitive abilities have in common (the operational definition of $g$ ). That is, tests of spatial ability correlate moderately with tests of verbal ability, and memory tests correlate with spatial and verbal tests, although more modestly. Factor analysis (an unrotated first principal component) creates a composite that weights each test by its overall correlation with all other tests. This vector of weights, called factor loadings, correlates with the heritabilities of the tests, that is, high $g$-loaded tests are the most heritable. In addition, $g$ loadings correlate with other biological and psychological processes, using a technique called the 'method of correlated vectors', which supports the validity of $g^{5}$.

Although $g$ is one definition of intelligence, intelligence has so many other connotations that it has been suggested that the word be avoided in scientific discussion ${ }^{5}$. Intelligence tests such as the individually administered Wechsler tests, which are widely used for clinical purposes, assess diverse abilities such as spatial ability (for example, making multicoloured blocks match a two-dimensional design), verbal ability (for example, vocabulary), speed of processing (for example, matching digits to symbols), memory (for example, memorizing a sequence of digits) and reasoning (for example, identifying what is missing in a picture). Rather that weighting these tests by their contribution to $g$, intelligence tests weight each test equally by summing them to create a composite score known as IQ, which is standardized to have a mean of 100 and a standard deviation of 15 . Nonetheless, IQ scores correlate highly $(r \approx 0.80)$ with $g$ scores derived from factor analysis.

these tests could investigate whether genetic correlations are also close to 1 between these modular measures and general processes ${ }^{21}$. As with many unanswered questions about genetics and cognitive abilities, clearer answers will emerge when specific genes are identified. In this case, the question is whether genes associated with $g$ are associated with a single general cognitive process, or with most modular processes, or with specific subsets of modular processes.

\section{Finding the genes for $g$}

The most far-reaching implications for science, and perhaps for society, will come from identifying genes responsible for the heritability of $g-$ not rare single-gene mutations that cause mental retardation, but QTLs that contribute probabilistically to individual differences in the normal variation in $g$.

Such loci are 'polymorphic'; that is, there are at least two, and often many more, variant forms of the gene in the population. These variants originally arise by mutations that change the actual coding sequence of a gene, thus producing a slightly different form of the encoded protein, or that affect the regulatory parts of a gene, thus affecting when and where the gene is switched on and protein produced. Both types of variation could contribute to the heritability of $g$.

Presumably, genes that are active in the brain ('expressed' in the brain in genetic terminology) are involved in specifying $g$, but with 30,000 or so genes known to be expressed in the brain this hardly helps to narrow the field. A small handful of genetic associations with behaviour have been found so far. The first definite associations of QTLs with behaviour have emerged in the area of cognitive disabilities, namely, dementia (an association with the apolipoprotein E gene) and reading disability (which has been linked to a region on the short arm of chromosome 6). Associations of $g$ with identified polymorphic segments of DNA on the genetic map (DNA markers) have begun to be reported ${ }^{22}$. As a result of the progress made in mapping the human genome, it is now becoming feasible to carry out genome scans using association approaches involving several thousand closely spaced DNA markers. These have the power to detect and locate the kind of genes that are likely to contribute to $g$, that is, multiple genes of small effect. The initial results of a systematic genome scan of thousands of DNA markers reported several replicated QTL associations ${ }^{23}$. The massive effort needed to genotype thousands of DNA markers for large numbers of subjects is daunting and replication is needed to eliminate false positive results. However, optimism about this approach has been fuelled by the promise of 'SNPs on chips' single nucleotide polymorphisms (SNPs) formatted as microarrays of oligonucleotide primers on solid substrates that can quickly genotype thousands of DNA markers for an individual. So far, such microarrays have been most useful in studies of gene expression and there remain technical difficulties to be overcome before they can be routinely used to genotype SNPs for large samples.

I have no doubt that genes associated with $g$ will be identified, although how much of the genetic variance will be accounted for by individual genes is uncertain. This is because the magnitude of the effects of genes in multiple gene systems is not yet known for $g$ or for any other trait or disorder controlled by a number of different genes (also known as complex traits and polygenic disorders). It is likely that the average size of effect of each individual gene is small for complex traits - 
perhaps individual genes on average will account for less than 1 per cent of the variance, with few effect sizes greater than 5 per cent, and a long tail of small effects extending to genes of such small effect that they may never be detected. If genes interact with each other they will be more difficult to identify because interactive combinations of genes would need to be found rather than individual genes. Fortunately, genetic effects on $g$ seem to be largely additive. Despite the formidable challenges of trying to find genes of small effect, I predict that most of the heritability of $g$ will be accounted for eventually by specific genes, even if hundreds of genes are needed to do it.

So what are the scientific and social implications of finding genes that influence $g ?$

\section{Implications for science}

One of the first tasks is to localize the specific DNA differences responsible for associations between DNA markers and $g$. Finding the locations of the genes involved will be greatly aided by several developments. The first will be the completion of the entire DNA sequence of the human genome, which is expected from the Human Genome Project during the next two years; the second is the recent intensive effort to identify hundreds of thousands of DNA variations among individuals, which will make it possible to pinpoint functional variants; and the third is the effort underway to map patterns of gene expression, which will indicate which genes are expressed in any given brain region.

The ultimate scientific goal is not just finding the genes but understanding how they function, and this is an even more challenging task. Functional genomics, as this aspect of genetics is called, is usually discussed in terms of molecular biological analyses of cell function. For example, studies of coordinated spatial and temporal patterns of gene expression using DNA chips containing detectors for thousands of genes - like functional imaging at a cellular level - will make major contributions to the study of gene function. However, other levels of analysis are also important in understanding how genes work. A behavioural level of analysis will also contribute to functional genomics, for example, by means of psychological theories of cognitive processing ${ }^{6}$ and by investigating interactions and correlations between individuals and their environment ${ }^{24}$. For instance, psychological theories suggest how different components of information processing are related and the role of genes in these cognitive systems can be examined. Such top-down strategies can yield just as important information as a bottom-up molecular approach in which the products of these genes are studied at a cellular level of analysis. As an antidote to the tendency to define functional genomics at the cellular level of analysis, the phrase 'behavioural genomics' has been proposed ${ }^{25}$ (see Fig. 2). Indeed, behavioural genomics may pay off more quickly than other levels of analysis in terms of prediction, diagnosis, therapy and intervention in relation to behavioural disorders and normal behavioural variation.

The brain is clearly where bottom-up molecular levels of analysis will eventually meet top-down behavioural analysis. Studies of brain functioning, as assessed by neuroimaging for example, will foster this integration. For humans, the expense of neuroimaging restricts sample sizes and, for this reason, differences between individuals are rarely considered. Mouse models will be valuable, especially given current large-scale behavioural screens of mice treated with mutagens. Finding genes associated with $g$ and other cognitive abilities and disabilities in humans will provide discrete windows through which brain pathways leading from genes to complex cognitive processes of learning and memory can be observed using animal models. Although learning and memory are the focus of much animal research in cognitive neuroscience, commonalities across cognitive processes that are indicative of $g$ have not yet been explored using animal models. The main focus at present is the study of synaptic connections between brain neurons and how they may be altered as an animal (or a person) learns or lays down a memory. The multivariate genetic results mentioned earlier lead to the hypothesis that, even at the cellular level, for example in connection with synaptic alterability, most genes will have broad effects on cognitive functioning rather than isolated effects on individual modules. That is, the same genes will affect many different brain structures and cellular processes. Although research on synaptic mechanisms oflearning and memory is leading the way in genetic research in cognitive neuroscience ${ }^{26}$, investigators have used gene knock-outs, in which a gene is altered so that it is no longer expressed, rather than studying naturally occurring genetic variation that might underlie individual differences in learning, memory and cognitive abilities. It is possible, but not inevitable, that a gene identified as being involved in learning and memory in gene knock-out experiments may also turn out to contribute to individual differences in $g$ when naturally occurring differences in the gene are identified.

The scientific impact of finding genes associated with $g$ will not be limited to cognitive neuroscience - it will affect all aspects of behavioural research. Perhaps some day behavioural and social scientists will routinely collect DNA using cheek swabs (where no blood is needed) in order to investigate, or at least control for, genes associated with $g$, as is happening now in research on dementia and its only known risk factor, the gene for apolipoprotein E. Even if hundreds of genes contribute to the heritability of $g$, finding genes associated with $g$ will make it possible to investigate long-standing scientific issues with much greater precision. For example, in relation to the finding that heritability of $g$ increases during development, are there additional genes associated with $g$ later in life, or do the same genes have greater effects as time progresses? What are the mechanisms by which gene-environment interactions and correlations emerge? Will the same genes affect different cognitive abilities and modular measures of brain function?

In terms of treatment-related research, finding genes associated with $g$ is likely to lead to gene-based diagnoses and treatment programmes for mild mental retardation, and clarification of its overlap with learning disabilities. Gene-based classification of disorders may bear little resemblance to our current symptom-based diagnostic systems. Indeed, from a QTL perspective, common disorders may be the quantitative extreme of normal genetic variation rather than qualitatively different. The most exciting prospect is for secondary prevention - if DNA analysis can be used to predict genetic risk for an individual, this might offer the hope of intervention before disorders create cascades of complications. The exemplar is phenylketonuria (PKU), a disorder that is due to a defect in a single gene. This leads to mental retardation unless it is detected early in life and dietary intervention is used to ameliorate its effects on the developing brain.

Perhaps the greatest implication for science is that the functional genomics of $g$ and other complex traits will serve as an integrating force across diverse disciplines, with DNA as the common denominator, opening up new scientific horizons for understanding behaviour.

\section{Implications for society}

At the outset, it should be emphasized that no policies necessarily follow from finding genes associated with $g$, because policy involves values. For example, finding genes associated with $g$ does not mean that we ought to put all of our resources into educating the brightest children. Depending on our values, we might worry more about children falling off the low end of the normal distribution in an increasingly technological society, and decide to devote more public resources to those in danger of being left behind. For example, all citizens need to be computer literate so that they will not be left on the shore while everyone else is surfing the World-Wide Web. There is much room for values here because these issues involve a complex balancing act among the rights and responsibilities of society, parents and 
children. The only thing that seems completely clear is that nothing will be gained by ignoring the issue and pretending that $g$ does not have a significant genetic component. As is always the case, advances in science create new challenges; we should be alert to such possibilities so that we can position ourselves to maximize the gains and minimize the pains of new discoveries. Recommended reading is an analysis of the ethics of the genetics of $g$ written by an ethicist and molecular geneticist ${ }^{27}$.

Will a DNA chip for $g$ make the 1997 science fiction film GATTACA, in which individuals are selected for education and employment on the basis of their DNA, come true? After childhood, no DNA chip can predict $g$ as well as an IQ test given to that individual, because IQ tests measure the consequences of environmental influences as well as genetic ones. I think it is more likely that educators and employers interested in $g$ will continue to use IQ tests and achievement tests to select individuals on the basis of what they can do, rather than using a DNA chip to estimate what they could have done. However, such a DNA chip might be used in education to consider how far children are fulfilling their genetic potential or to prescribe different training programmes.

DNA chips for $g$ might be used for prenatal testing, for example, in the selection of embryos for in vitro fertilization. But this seems unlikely, because few embryos are available to choose from and there are many important genetic diseases to screen out. What about parents who want to use DNA chips for $g$ in order to select egg or sperm donors, because such a chip might provide better estimates of genetic potential than phenotypic tests of the donors? Is it possible that there are parents who would use DNA chips for $g$ prenatally for eugenic purposes?

Will DNA chips for $g$ be used for postnatal screening to enable interventions that avoid risks or enhance strengths? For decades we have screened newborns for PKU because a relatively simple dietary intervention exists that prevents its damage to the developing brain. If similarly low-tech and inexpensive interventions such as dietary changes could make a difference for some $g$ genotypes, parents might want to take advantage of them even if the QTL accounts for only a small amount of variance. Expensive high-tech genetic engineering in regard to behavioural traits is unlikely to happen for a long time - it is proving very difficult even for the single-gene disorders, and it will be many orders of magnitude more difficult and less effective for complex traits influenced by many genes.

A more general concern involves group differences, such as average differences between classes and ethnic groups. As genes are found that are associated with differences among individuals within groups, the genes will inevitably be used to make comparisons between groups. Although such comparisons will not be straightforward because the causes of individual differences within groups are not necessarily related to the causes of average differences between groups, the societal implications of such research need to be anticipated. To keep this issue in perspective, it should be emphasized that average differences in $g$ between groups are small compared with the range of individual differences within groups. Perhaps people will become less preoccupied with average differences between groups when DNA chips make it possible to focus on individuals.

Another general concern is that knowledge about the importance of genetics might change attitudes - for example, attitudes of parents about the malleability of their children's cognitive ability. If there are parents who do not recognize genetic limits to their children's ability, it might actually be useful for them to have a more realistic view, so that their children's failures are not interpreted as simple motivational failures. Do parents matter? They do indeed. And not just because of their genes. Although the genetic research discussed earlier indicates that parents do not mould their children environmentally to be similar to them in terms of $g$, genetic research on $g$ is mute about much that parents offer their children as teachers and models independent of the parents' $g$. Moreover, as Judith Harris concludes in her book, The Nurture Assumption: "We may not hold their tomorrows in our hands but we surely hold their todays, and we have the power to make their todays very miserable."16

The most general fear is that finding genes associated with $g$ will undermine support for social programmes because it will legitimate social inequality as 'natural.' The unwelcome truth is that equal opportunity will not produce equality of outcome because people differ in $g$ in part for genetic reasons. When the US founding fathers declared that all men are created equal they did not mean that all people are identical, but rather that they should be equal before the law. Democracy is needed to ensure that all people are treated equally despite their differences. On the other hand, finding heritability or even specific genes associated with $g$ does not imply that $g$ is immutable. Indeed, genetic research provides the best available evidence that non-genetic factors are important in the development of individual differences in $g$. PKU provides an example that even a single gene that causes mental retardation can be ameliorated environmentally.

'There is no gene for the human spirit' is the subtitle of the film GATTACA. It embodies the fear lurking in the shadows that finding genes associated with $g$ will limit our freedom and our free will. In large part such fears involve misunderstandings about how genes affect complex traits like $g^{28}$. Finding genes associated with $g$ will not open a door to Huxley's brave new world where babies are engineered to be alphas, betas and gammas. The balance of risks and benefits to society of DNA chips for $g$ is not clear - each of the problems identified in this section could also be viewed as a potential benefit, depending on one's values. What is clear is that basic science has much to gain from functional genomic studies of brain functions related to learning and memory. We need to be cautious and to think about societal implications and ethical issues. But there is also much to celebrate here in terms of the increased potential for understanding our species' unparallelled ability to think and learn.

Robert Plomin is at the Institute of Psychiatry, King's College London, DeCrespigny Park, Denmark Hill, London SE5 8AF, UK.

e-mail:r.plomin@iop.kcl.ac.uk

1. Plomin, R., Owen, M. J. \& McGuffin, P. Science 264, 1733-1739 (1994).

2. Gottfredson, L. S. Intelligence 24, 13-23 (1997).

3. Gould, S. J. The Mismeasure of Man (W. W. Norton, New York, 1996).

4. Carroll, J. B. Intelligence 21, 121-134 (1995).

5. Jensen, A. R. The g factor: The Science of Mental Ability (Praeger, Westport, 1998).

6. Mackintosh, N. J. IQ and Human Intelligence (Oxford University Press, Oxford, 1998).

7. Herrnstein, R. J. \& Murray, C. The Bell Curve: Intelligence and Class Structure in American Life (The Free Press, New York, 1994).

8. Brody, N. Intelligence (Academic Press, New York, 1992).

9. Snyderman, M. \& Rothman, S. The IQ Controversy, the Media and Publication (Transaction, New Brunswick, NJ, 1988).

10. Bouchard, T. J. Jr \& McGue, M. Science 212, 1055-1059 (1981).

11. Plomin, R., DeFries, J. C. McClearn, G. E. \& Rutter, M. Behavioral Genetics (W. H. Freeman, New York, 1997).

12. McGue, M., Bouchard, T. J., Iacono, W. G. \& Lykken, D. T. in Nature, Nurture, and Psychology (eds Plomin, R. \& McClearn, G. E.) 59-76 (American Psychological Association, Washington, DC, 1993).

13. McClearn, G. E. et al. Science 276, 1560-1563 (1997).

14. Flynn, J. Am. Psychol. 54, 5-20 (1999).

15. Duyme, M., Dumaret, A.-C. \& Tomkiewicz, S. Proc. Natl Acad. Sci. USA 96, 8790-8794 (1999).

16. Harris, J. R. The Nurture Assumption: Why Children Turn Out the Way They Do (The Free Press, New York, 1998).

17. Rowe, D. C. \& van den Oord, E. J. C. G. Child Dev. (in the press).

18. Plomin, R. Genetics and Experience: The Interplay Between Nature and Nurture (Sage Publications, Thousand Oaks, CA, 1994).

19. Plomin, R. \& DeFries, J. C. Sci. Am. May, 62-69 (1998). 20. Petrill, S. A. Curr. Directions Psychol. Sci. 6, 96-99 (1997).

21. Kosslyn, S. \& Plomin, R. in Psychiatric Neuroimaging Strategies: Research and Clinical Applications (eds Dougherty, D., Rauch, S. L. \& Rosenbaum, J. F.) (American Psychiatric Press, Washington, DC, in the press)

22. Chorney, M. J. et al. Psych. Sci. 9, 1-8 (1998). 23. Fisher, P. J. et al. Hum. Mol. Genet. 8, 915-922 (1999). 24. Plomin, R. \& Rutter, M. Child Dev. 69, 1221-1240 (1998). 25. Plomin, R. \& Crabbe, J. C. Psychol. Bull. (in the press). 26. Migaud, M. et al. Nature 396, 433-439 (1998)

27. Newson, A. \& Williamson, R. Bioethics 13, 327-342 (1999). 28. Rutter, M. \& Plomin, R. Br. J. Psychiat. 171, 209-219 (1997).

Acknowledgements. Preparation of this paper and the QTL research on $g$ is supported by a grant from the US National Institute of Child Health and Human Development. The paper profited from review of an earlier version by R. Arden, S. BaronCohen, I. Craig, P. Dale, I. J. Deary, J. C. DeFries, L. S. Gottfredson, F. Happé, J. Rich Harris, C. Hughes, D. Lubinski, P. McGuffin, A. Newson, T. G. O'Connor, M. J. Owen, S. A. Petrill, K. J. Saudino, L. A. Thompson and I. D. Waldman. 